\title{
Horace Barlow: a vision scientist for the ages
}

\author{
Terrence J. Sejnowski ${ }^{1,2}$
}

(c) Springer-Verlag GmbH Germany, part of Springer Nature 2021

Horace Barlow, doyen of the visual sciences, died on July 5,2020 at the age of 98 . His research illuminated many fundamental issues in vision and his efficient coding hypothesis was a beacon for vision research. His probing mind and playful spirit influenced the questions asked by a generation of vision researchers, bringing us closer to understanding how we see.

As a student, Barlow was on track for a medical degree. Through good fortune and research studentships from the Rockefeller Foundation and the Medical Research Council, his talents for scientific inquiry became apparent. His career blossomed in Cambridge after 1950 and he later moved to UC Berkeley in 1964, where, along with Gerald Westheimer and Bill Levick, he attracted and trained many of today's leading visual physiologists and psychophysicists. He moved back to Cambridge in 1973, where his insights into the neural basis of visual perception deepened.

Early in his career, Barlow produced a series of singleauthored landmark studies that are in textbooks. One study from that period asked the question: How sensitive is the retina to detecting photons at absolute threshold (Barlow 1956)? Based on elegant experimental design and careful analysis he concluded that the absorption of a single photon could excite a rod photoreceptor in the human retina. However, because of spontaneous isomerization of rhodopsin and other sources of noise, the coincidence of events in multiple excited rods are needed to evoke the sensation of a light flash.

At Berkeley, Barlow discovered neurons in the primary visual cortex tuned for stereoscopic vision. Hubel and Wiesel had reported neurons that receive visual input from both eyes. Barlow found that the best responses occurred not

Terrence J. Sejnowski

terry@snl.salk.edu

1 Computational Neurobiology Laboratory, The Salk Institute for Biological Studies, La Jolla, CA 92037, USA

2 Division of Biological Sciences, University of California, San Diego, La Jolla, CA 92093, USA when the visual stimulus was at the same angle from the fovea in the two eyes, but at an offset, or binocular disparity (Barlow et al. 1967). Different cells were tuned to different disparities, corresponding to different depth planes.

Barlow was influenced early in his career by Norbert Wiener's book on Cybernetics (1948) and Claude Shannon's article on A Mathematical Theory of Communication (1948), which later inspired his insights into neural coding. He was a founder of the Ratio Club in 1949, focused on cybernetics, whose members were an eclectic group of young neurobiologists, engineers, mathematicians and physicists, including Alan Turing, many of whom went on to highly prominent careers. The central tenet of Barlow's efficient coding hypothesis (Barlow 1959, 1961) was that brains represent information in the world by a minimum number of spikes. His hypothesis that the output of the retina reduced redundancy in visual input provided an elegant explanation for why the receptive fields of ganglion cells had centers and surrounds of opposite polarity, which minimized output spikes for uniformly illuminated visual patches.

Barlow further hypothesized that the simple cells discovered by Hubel and Wiesel in the visual cortex, which resembled Gabor functions, were a sparse and efficient representation for natural scenes (Barlow 1983). At the time, there was no way to confirm his hypothesis, but the development of computational methods for sparse coding (Olshausen and Field 1996) and Independent Component Analysis (Bell and Sejnowski 1997) showed that he was right. Localized Gabor functions are a sparse and efficient basis for natural scenes, but not for unnatural images like this printed page.

In another influential paper (Barlow 1972) entitled Single units and sensation: A neuron doctrine for perceptual psychology?, Barlow applied his ideas to how neurons represent complex objects at higher levels of the visual cortex, where neural responses are more selective and activity in the neural population sparser than at the early stages. He argued against "pontifical cells," single neurons that code the percept of unique objects, which was the basis for the so-called "grandmother cell theory" that had taken hold in the vision community. He argued instead that an object should be represented 


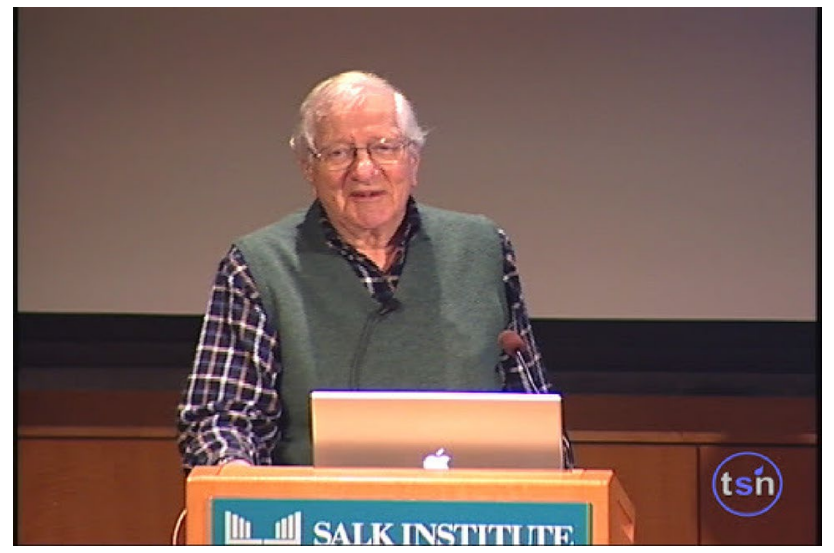

Horace Barlow delivering The Francis Crick Nobel Lecture on Friday, May 15, 2009 on "Selectivity and Symmetry in the Function of the Cerebral Cortex" Courtesy of The Science Network

by a population of "cardinal cells," each representing unique combinations of features of the object, together forming a distributed invariant representation of the object.

Barlow lived to see a revolution in computational models of cortical processing based on the architecture of the visual cortex (Sejnowski 2020). Deep learning networks trained to recognize objects in images, mimicking the progression of response properties of neurons in the visual hierarchy, have what looks like "cardinal cells" at their highest levels (Yamins et al. 2014). This corroborates Barlow's conjecture that his cardinal cells could support the invariant perception of objects.

Barlow's long life straddled a period of visual neuroscience that was dominated by the single unit technique. Theories are driven by techniques, and the single unit recording technique focused theory on single neurons. The grandmother cell theory was in essence the microelectrode theory of brain function. Barlow's clear thinking allowed him to see beyond single neurons without having the techniques that we have today for recording simultaneously from many thousands of neurons.

Another influential concept introduced by Barlow was that of a suspicious coincidence between independent features (Barlow 1985). This was the logical next step beyond his earlier insight that the brain was in the business of building an efficient statistical model of the sensory stream. A suspicious coincidence is an event that is statistically unlikely, something that arouses the brain's interest, both for short-term investigation and long-term recall. This concept is related to surprise, which transcends vision and invokes neuromodulatory systems. It also connects vision to memory systems since we tend to remember what is unusual better than what is usual (Barlow 1990).
A hundred years from now, Horace Barlow will be remembered for his pioneering physiological studies and for his penetrating insights into the foundations of vision in the twentieth century, much as Helmholtz is today remembered for his seminal discoveries on visual perception in the nineteenth century. Barlow guided our thinking about vision during a heroic period of biological discovery, and continues to influence vision science in the twenty-first century.

Editor's note: Horace Barlow has been on the editorial board of Biological Cybernetics since it was founded in 1961 as Kybernetik.

\section{References}

Barlow HB (1956) Retinal noise and absolute threshold. J Opt Soc Am 46:634-639

Barlow HB (1959) Sensory mechanisms, the reduction of redundancy, and intelligence. The mechanisation of thought processes. H.M.S.O, London, pp 535-539

Barlow H (1961) Possible principles underlying the transformation of sensory messages. In: Sensory communication. MIT Press

Barlow HB (1972) Single units and sensation: a neuron doctrine for perceptual psychology?. Perception 1(4):371-394

Barlow HB (1983) Understanding natural vision. In: Braddick OJ, Sleigh AC (eds) Physical and biological processing of images. Vol. 11 of Springer series in information sciences. Springer, Berlin, pp 2-14

Barlow HB (1985) Cerebral cortex as model builder. In: Rose D, Dobson VG (eds) Models of the visual cortex. Wiley, New York, pp $37-46$

Barlow HB (1990) Conditions for versatile learning, Helmholtz's unconscious inference, and the task of perception. Vision Res 30:1561-1571

Barlow H, Blakemore C, Pettigrew J (1967) The neural mechanisms of binocular depth discrimination. J Physiol 193:327-342

Bell AJ, Sejnowski TJ (1997) The 'Independent Components' of natural scenes are edge filters. Vis Res 37:3327-3338

Olshausen BA, Field DJ (1996) Emergence of simple-cell receptive field properties by learning a sparse code for natural images. Nature 381:607-609

Sejnowski TJ (2020) The unreasonable effectiveness of deep learning in artificial intelligence. Proc Natl Acad Sci USA 48:30033-30038

Shannon CE (1948) A mathematical theory of communication. Bell Syst Tech J 27(379-423):623-656

Wiener N (1948) Cybernetics. Wiley, New York

Yamins DL, Hong H, Cadieu CF, Solomon EA, Seibert D, DiCarlo JJ (2014) Performance-optimized hierarchical models predict neural responses in higher visual cortex. Proc Natl Acad Sci USA 111:8619-8624

Publisher's Note Springer Nature remains neutral with regard to jurisdictional claims in published maps and institutional affiliations. 